\title{
Rugby in Rio in 2016!
}

\section{Lars Engebretsen, Kathrin Steffen}

Recently, rugby was voted in as one of the new summer Olympic sports and will appear in Rio in 2016-for many rugby fans, not a minute too soon; for the Olympic sports medicine community, another challenge on how to implement prevention programmes without changing the game itself.

We celebrate the inclusion of rugby into the Olympic family by dedicating this issue to rugby. Several papers on rugby injuries and their prevention are here selected to broaden focus on this sport that is deep-rooted in the Commonwealth and on the rise in many other countries.

Rugby has long had good sports medicine service. A PubMed search in December 2009 revealed 2751 papers on rugby and injuries and 654 on rugby, injuries and prevention - the majority of these were either studies in epidemiology or conclusions such as 'and prevention studies must be carried out,' but the fact is that elite rugby has been able to decrease the number of injuries through rule changes and other preventive measures. In this issue of Injury Prevention and Health Protection (IPHP), five papers highlight epidemiology, risk factors and prevention strategies through rule changes and improved equipment. We think rugby can help us lead the way for similar actions by other Olympic sports.

One sport clearly in need of a substantial prevention programme seems to be Olympic-style wrestling. A report from an Iranian group published in this issue ${ }^{1}$ (see page 168) shows that catastrophic injuries are rare, 2/100 000 wrestlers per year. However, the sport is extremely popular in countries like Iran and other East Asian countries, and needs public focus on injury prevention. In their paper, the authors attempt to identify major risk factors for catastrophic wrestling injuries, which for the future will play an essential role in affecting rule changes and thereby hopefully injury prevention, too.

In the current issue, we also present news on risk-taking behaviour in skiing/ snowboarding to prevent injuries in these sports, ${ }^{2}$ (see page 204) data from a cohort of elite rowers, ${ }^{3}$ (see page 207) as well as results from a prospective cohort study on physical activity and sports related injuries in children. ${ }^{4}$ (see page 158) These papers are important contributions to sports medicine which should help sports medicine clinicians and researchers in implementing current knowledge on injury prevention.

As one of their tasks, the International Olympic Committee (IOC) has decided to financially support established research centres that have demonstrated clinical, educational and research expertise in elite sports. In September 2009, the IOC announced that four sports medicine centres were chosen as 'IOC research centres for IPHP.' The tasks for the centres, located in Australia, Canada, Norway and South Africa, will be: - establishing long-term research programmes on injury and disease prevention (including studies on basic epidemiology, risk factors, injury mechanisms and intervention);

- consequences of sports injuries and sports-related illnesses;

- pathophysiology, risk factors and injury mechanisms;

- methods for sports injury and disease prevention;

- fostering collaborative relationships with individuals, institutions and organisations to improve athletes' health;

- implementing (and collaborating with) applied, ongoing and novel research and development within the framework and long-term strategy of the IOC;

- setting up knowledge translation mechanisms to share scientific research results with the field throughout the Olympic Movement and sports community, and to convert these results into concrete action to protect the health of the athletes;

The IOC will work with these centres for the coming 4 years to increase research and find ways of implementing the results.

Br J Sports Med 2010;44:157

doi:10.1136/bjsm.2010.71555

\section{REFERENCES}

1. Kordi R, Akbarnejad A, Wallace AW. Catastrophic injuries in the Olympic styles of wrestling in Iran. Br Journal Sport Med 2010;44:168-174

2. Ruedl G, Pocecco E, Sommersacher R, et al. Factors associated with self reported risk taking behaviour on ski slopes. Br Journal Sports Med 2010;44:204-206

3. Wilson F, Gissane C, Simms C, et al. A 12 month prospective cohort study of injury in international rowers. Br Journal Sports Med 2010;44:207-214

4. Verhagen E, van Mechelen M. Sport for all, injury prevention for all. Br Journal Sports Med 2010:44:158 